\title{
Event-triggered Control for Discrete-Time Systems
}

\author{
Alina Eqtami, Dimos V. Dimarogonas and Kostas J. Kyriakopoulos
}

\begin{abstract}
In this paper, event-triggered strategies for control of discrete-time systems are proposed and analyzed. Similarly to the continuous-time case, the plant is assumed input-tostate stable with respect to measurement errors and the control law is updated once a triggering condition involving the norm of a measurement error is violated. The results are also extended to a self-triggered formulation, where the next control updates are decided at the previous ones, thus relaxing the need for continuous monitoring of the measurement error. The overall framework is then used in a novel Model Predictive Control approach. The results are illustrated through simulated examples.
\end{abstract}

\section{INTRODUCTION}

Traditional approaches to sampling for feedback control involve a time-periodic decision ruling. However, this might be a conservative choice. In some cases, equidistant sampling can be prohibitive to attain certain goals. For example, the issues of limited resource and insufficient communication bandwidth for decentralized control of large scale systems, or even the case of inadequate computation power for fast systems, are problems that often have to be dealt with. A recent approach is to sample only when is needed. Even though we need to relax the periodicity for computation of the control law, we still need to preserve necessary properties of the system, such as stability and convergence. It is therefore of great interest to build mechanisms for sampling that do not rely on periodicity or time-triggering techniques. A comparison of time-driven and event-driven control for stochastic systems favoring the latter can be found in [2]. As a result, in recent years the issue of event-driven feedback and sampling, has been developed. The key attribute of these approaches, is that the decision for the execution of the control task is not made ad-hoc, but it is based on a certain condition of the state of the system.

In [20], the control actuation is triggered whenever a certain error norm becomes large enough to overtake the norm of the state of the plant. The nominal system is assumed to be Input-to-State stable [19], with respect to measurement errors and then, tools from perturbation analysis of nonlinear systems are used in order to analyze the performance of the event-driven system. The results revealed that the proposed scheme can maintain desired levels of performance. In a similar context as [20], the authors in [13] have resorted to an event-driven policy for sensor/ actuator networks, which

Alina Eqtami and Kostas J. Kyriakopoulos are with the Control Systems Lab, Department of Mechanical Engineering, National Technical University of Athens, 9 Heroon Polytechniou Street, Zografou 15780, Greece $\{a l i n a, k k y r i a @ m a i l . n t u a . g r\}$. Dimos V. Dimarogonas is with the Laboratory for Information and Decision Systems, Massachusetts Institute of Technology, Cambridge, MA 02139, U.S.A. $\{$ ddimar@mit.edu\}. resulted in less energy consumption. Event-driven strategies for multi-agent are presented in [4], [5], while stochastic event-triggered strategies regarding sensing and actuation for networked control systems have been stated in [18]. More recently, a new concept of event-predictive control for energy saving of wireless networked control systems has appeared, [8]. An alternative approach to event-driven control, for perturbed linear systems can be found in [6]. In the case of event-triggered feedback nonlinear systems a different approach can be seen in [22]. Under the proposed scheme the average sampling period has been elongated, compared to previous works.

Event-driven techniques require the constant measurement of the current state of the plant, in order to decide when the control execution must be triggered. In the case of selftriggered control [23], [1], only the last state measurement needs to be known for determining the next time instant where the state must be measured again so that the control law is computed and the actuators are updated. A first attempt has been made for linear systems in [21] and recently for systems with finite-gain $\mathcal{L}_{2}$ stability. Some particular classes of nonlinear systems, namely state-dependent homogeneous systems and polynomial systems, under self-triggered policy have been presented in [1]. Related works can be found in [12], [13], [16], [15].

All of the approaches, previously mentioned, have been performed in the continuous-time frame. The contribution of this paper is to show how the event-triggered, as well as the self-triggered techniques can be implemented over the discrete-time frame. The main assumption used for the event-triggered policies, is the Input-to-State property of the plant. For general nonlinear discrete-time systems the ISS characterization was first introduced in [10], and later refined in [11]. For sampled-data systems notions of ISS stability can be found in [17], while in [7] minimal ISS gains and transient bounds are given for discrete nonlinear systems.

The purpose of this paper is to study how the eventtriggered as well as the self-triggered control can be reformed in the case of discrete-time systems. The remainder of this paper is comprised of five Sections. In Section II we provide an equivalent relation of general discrete-time systems under event-triggered formulation. Triggering conditions under event and self-trigger policies for linear discrete systems are provided in Section III. Following similar procedure-as in the continuous-time context we present another approach to computing inter-execution time for discrete-time systems, in Section IV. Motivated by the fact that Model Predictive Control schemes are computationally demanding algorithms, we investigate in Section V the use of event-triggered control 
for relaxing these computational needs. Section VI, presents some simulation results in order to show the efficiency of the proposed schemes. We conclude with some brief remarks on Section VII.

\section{EVENT-TRIGGERED CONTROL}

In this section we are going to introduce the eventtriggered formulation for a general nonlinear system as in [20], in its discrete-time counterpart, and we will state the general event-triggering rule for sampling.

Consider a control system in the discrete time-domain of the general form

$$
x(k+1)=f(x(k), u(k))
$$

where $x \in \mathbb{R}^{n}$ is the state, $x(k+1) \in \mathbb{R}^{n}$ is the successor state, and $u \in \mathbb{R}^{m}$ are the control values, for each time instant $k \in \mathbb{Z}_{+}$. The vector field $f: \mathbb{R}^{n} \times \mathbb{R}^{m} \rightarrow \mathbb{R}^{n}$ is assumed to be continuous. Also assume without loss of generality, that the origin is an equilibrium point for (1), i.e., $f(0,0)=0$.

Let the system (1) be continuously stabilizable by a continuous feedback law of the form $u(k)=w(x(k))$, with $w: \mathbb{R}^{n} \rightarrow \mathbb{R}^{m}$. Then, system (1) is ISS-stabilizable with respect to measurement errors $e(k)$, i.e., there is feedback control law, $p: \mathbb{R}^{n} \rightarrow \mathbb{R}^{m}$ of the form $u(k)=p(x(k)+e(k))$ that renders the closed-loop system

$$
x(k+1)=f(x(k), p(x(k)+e(k)))
$$

Input-to-State (ISS) stable with respect to measurement errors $e(k),[11]$. As in classic Lyapunov theory a system that is ISS-stable, admits an ISS-Lyapunov function, [14]. A continuous function $V: \mathbb{R}^{n} \rightarrow \mathbb{R}_{\geqslant} 0$ is an ISS-Lyapunov function for the system (2) if there exist $\mathcal{K}_{\infty}$ functions $\alpha_{1}, \alpha_{2}$, such that

$$
\alpha_{1}(\|x\|) \leq V(x) \leq \alpha_{2}(\|x\|) \quad \forall x \in \mathbb{R}^{n}
$$

and for some $\alpha$ that is class $\mathcal{K}_{\infty}$ function, and $\gamma$ that is class $\mathcal{K}, V(x)$ also satisfies

$$
\begin{array}{r}
V(f(x(k), p(x(k)+e(k))))-V(x(k)) \leq \\
-\alpha(\|x(k)\|)+\gamma(\|e(k)\|)
\end{array}
$$

Assume that in the event-triggered setup, the control is updated at the discrete time instants

$$
k_{0}, k_{1}, k_{2}, \ldots
$$

The control law is defined as

$$
u(k)=p\left(x\left(k_{i}\right)\right), \quad k \in\left[k_{i}, k_{i+1}\right)
$$

i.e., it remains constant in the inter-execution interval. We assume that at the sampling instant $k_{i}, k_{i}>0$ the state variable vector $x\left(k_{i}\right)$ is available through measurement and provides the current plant information. Defining the state measurement error in this interval, as

$$
e(k)=x\left(k_{i}\right)-x(k), \quad k \in\left[k_{i}, k_{i+1}\right)
$$

the stabilizable feedback control law is now given by

$$
p\left(x\left(k_{i}\right)\right)=p(x(k)+e(k))
$$

with $k \in\left[k_{i}, k_{i+1}\right)$ and the closed-loop equation of system (1) becomes

$$
x(k+1)=f(x(k), p(x(k)+e(k)))
$$

System (7) remains ISS-stable, if $e(k)$ satisfies

$$
\gamma(\|e\|) \leq \sigma a(\|x\|)
$$

with $0<\sigma<1$. Invoking this rule into equation (4), it becomes

$$
V(f(x(k), p(x(k)+e(k))))-V(x(k)) \leq(\sigma-1) a(\|x\|)
$$

with $V$ still guaranteed to be decreasing. Hence, the control law should be updated as long as the condition

$$
\gamma(\|e\|) \leq \sigma a(\|x\|)
$$

holds.

Theorem 1: Consider the system (1) and assume that the previously presented assumptions hold. Then the control law (2) with the event-triggered ruling (10), asymptotically stabilizes the system to the origin.

\section{LINEAR DISCRETE-TIME SySTEMS}

Using the general notion for event-trigger policy, presented above, we specialize to discrete-time, time-invariant linear systems. In this case, the triggering policy is found to be also linear with respect to the state of the plant.

The system under consideration is

$$
x(k+1)=A x(k)+B u(k)
$$

where $A \in \mathbb{R}^{n \times n}, B \in \mathbb{R}^{n \times m}$ and the input $u(k) \in \mathbb{R}^{m}$ are defined in $k \in \mathbb{Z}_{+}$. The pair $(A, B)$ is considered to be stabilizable, which means that there exists a matrix $K$ so that the eigenvalues of $A+B K$ are inside the unit disc, then the system (11) is also ISS-stabilizable. ISS properties of linear discrete-time systems were provided in [11].

Input-to-State stabilizability of system (11), implies that there exists a stabilizing feedback control law $u(k)=$ $K(x(k)+e(k))$ where $e$ is the measurement error seen as a new input and, $K$ is an appropriate matrix, defined above. The compensated closed-loop system (11), is described by the equation

$$
x(k+1)=(A+B K) x(k)+B K e(k)
$$

System (12) admits a quadratic ISS-Lyapunov function of the form

$$
V(x)=x^{T} P x
$$

Function $V$ is considered to be positive-definite, radially unbounded and satisfies property (3) with $\alpha_{1}(r)=\lambda_{\min }(P) r^{2}$ and $\alpha_{2}(r)=\lambda_{\max }(P) r^{2}$, with $\lambda_{\min }(P), \lambda_{\max }(P)$ the smallest and the largest eigenvalue of matrix $P$, respectively. Given a symmetric and positive-definite matrix $Q$, let $P$ be the unique positive definite solution to

$$
(A+B K)^{T} P(A+B K)-P=-Q
$$


The difference of the ISS-Lyapunov function is

$$
\begin{aligned}
& V(x(k+1))-V(x(k))= \\
& -x^{T}(k) Q x(k)+2 x^{T}(A+B K)^{T} P B K e(k) \\
& +e^{T}(k) K^{T} B^{T} P B K e(k)
\end{aligned}
$$

Then, the property (4), holds with

$$
\begin{gathered}
\alpha(\|x\|)=\frac{1}{2} \lambda_{\min }(Q)\|x\|^{2} \\
\gamma(\|e\|)=\left(\frac{2\left\|(A+B K)^{T} P B K\right\|^{2}}{\lambda_{\min }(Q)}+\left\|K^{T} B^{T} P B K\right\|\right)\|e\|^{2}
\end{gathered}
$$

In an event-triggered formulation of system (12), with ISS-Lyapunov equation of the form (13) the control updates should be enforced as long as

$$
\|e\| \leq\left(4 \frac{\left\|(A+B K)^{T} P B K\right\|^{2}}{\sigma \lambda_{\min }^{2}(Q)}+2 \frac{\left\|K^{T} B^{T} P B K\right\|}{\sigma \lambda_{\min }(Q)}\right)^{-1}\|x\|
$$

with $0<\sigma<1$, holds. Thus (16) is the equivalent of (10) in the linear case. We also use the notation

$$
\mu=\left(4 \frac{\left\|(A+B K)^{T} P B K\right\|^{2}}{\sigma \lambda_{\min }^{2}(Q)}+2 \frac{\left\|K^{T} B^{T} P B K\right\|}{\sigma \lambda_{\min }(Q)}\right)^{-1}
$$

in the following paragraphs.

In the sequel, a result on the minimum time between two consecutive executions is presented for the linear case. We note here that non-trivial lower bounds on the inter-execution times, i.e. bounds strictly larger than one, are not suitable for the systems considered here due to their discrete time nature. A proposition providing sufficient conditions for non-trivial inter-execution times is given in the following paragraphs.

We consider how the state as well as the error evolve with time. In view of equation (6), the system described in equation (12) now becomes

$$
x(k+1)=A x(k)+B K x\left(k_{i}\right)
$$

where $k_{i}$ is the latest actuation update instant. We set the vector $c_{1}=B K x\left(k_{i}\right)=$ const., and thus the solution of (17) is

$$
x(k)=A^{k} x\left(k_{i}\right)+\sum_{j=0}^{k-1} A^{k-1-j} c_{1}
$$

It is straightforward to see, that the error at the next discrete time instant is given by $e(k+1)=x\left(k_{i}\right)-x(k+1)$. Thus, equation (12) with some manipulation becomes

$$
e(k+1)=(A+B K) e(k)+(I-A-2 B K) x\left(k_{i}\right)
$$

The solution of this linear nonhomogeneous equation, is given by

$$
e(k)=\sum_{j=0}^{k-1}(A+B K)^{k-1-j} c_{2}
$$

where we set $c_{2}=(I-A-2 B K) x\left(k_{i}\right)=$ const. and $e\left(k_{i}\right)=0$.
Define now the minimum $k=k^{*}$ that violates condition (16), i.e.,

$$
\begin{gathered}
k^{*}=\arg \min _{k \in \mathbb{N}}\left\{\left\|\sum_{j=0}^{k-1}(A+B K)^{k-1-j} c_{2}\right\| \geq\right. \\
\left.\mu\left\|A^{k} x\left(k_{i}\right)+\sum_{j=0}^{k-1} A^{k-1-j} c_{1}\right\|\right\}
\end{gathered}
$$

Proposition 2: Consider the system (12) and assume that (21) has a solution $k^{*}>1$ for all $k_{i}$. Then the event-triggered rule (16) is non-trivial, in the sense that it takes at least two steps for the next controller update.

Further investigation of the sufficient conditions given in the above Proposition is a topic of ongoing research.

\section{A. Self-triggered control}

Another view for finding sampling periods is the selftriggered formulation. Motivated by the corresponding selftriggered notion which was originally proposed by for the continuous-time systems, here we are going to provide their discrete analogues. Using this kind of implementation, inter-execution times are provided as in the event-triggered implementation, but in this case no continuous monitoring of the plant's state is required. We shall write the system (12) in a state-space representation by eliminating variable $x\left(k_{i}\right)$, while treating $e(k)$ as a new state variable:

$$
\left[\begin{array}{l}
x_{k+1} \\
e_{k+1}
\end{array}\right]=\left[\begin{array}{cc}
A+B K & B K \\
I-A-B K & I-B K
\end{array}\right]\left[\begin{array}{l}
x_{k} \\
e_{k}
\end{array}\right]
$$

We define $y=\left[x(k)^{T}, e(k)^{T}\right]^{T}$, and

$$
C=\left[\begin{array}{cc}
A+B K & B K \\
I-A-B K & I-B K
\end{array}\right]
$$

This system in a stack vector form becomes a linear homogeneous system, in particular it can be rewritten as

$$
y(k+1)=C y(k) \quad \text { with } \quad y_{0}=y_{k_{0}}
$$

The initial conditions of this system, at each sampling period, is $y_{k_{0}}=\left[x\left(k_{i}\right)^{T}, 0\right]^{T}$, and the solution of (22) is of the form

$$
y(k)=C^{k-k_{i}} y_{k_{0}}
$$

As in the general event-triggered formulation, the difference of the ISS-Lyapunov function must be negative, and an inequality of the form (10) must exist. As we saw in (16), at the linear case this inequality becomes also linear. In view of (16), while making little manipulations, this inequality can be re-written as

$$
\begin{aligned}
\|e(k)\|^{2}+\|x(k)\|^{2} & \leq \mu^{2}\|x(k)\|^{2}+\|x(k)\|^{2} \\
\|y(k)\|^{2} & \leq\left(1+\mu^{2}\right)\|\tilde{I} y(k)\|^{2}
\end{aligned}
$$

with $\tilde{I}=\left[\begin{array}{ll}I & 0\end{array}\right]$. Similarly to the derivation of Proposition 2 , define the minimum $k=k^{* *}$ that violates condition (23), i.e.,

$$
\begin{aligned}
k^{* *}= & \arg \min _{k \in \mathbb{N}}\left\{\left\|C^{k-k_{i}} y_{k_{0}}\right\|^{2} \geq\right. \\
& \left.\left(1+\mu^{2}\right)\left\|\tilde{I} C^{k-k_{i}} y_{k_{0}}\right\|^{2}\right\}
\end{aligned}
$$


We now can state the following result for the interexecution times in this formulation:

Proposition 3: Consider the system (12) and assume that (24) has a solution $k^{* *}>1$ for all $k_{i}$. Then the self-triggered rule (23) is non-trivial, in the sense that it takes at least two steps for the next controller update.

It is worth noting from equation (24), that only the current state of the plant is required to compute the next execution time of the control, thus at each time instant it is known when the next sampling time is going to take place.

\section{ANOTHER APPROACH TO COMPUTING THE INTER-EXECUTION TIMES}

In this section we propose another event-triggered strategy which follows a similar strategy with the approach in [22]. The approach is valid for a smaller class of nonlinear systems that satisfy stronger stability conditions. Recall

$$
x(k+1)=f(x(k), p(x(k)+e(k)))
$$

and assume that the following assumption holds:

Assumption 1: There exist positive constants $L, L_{1}, a, \gamma \geq 0$, a $C^{1}$ function $W: \mathbb{R}^{n} \rightarrow \mathbb{R}_{\geqslant 0}$, and $\mathcal{K}_{\infty}$ functions $\alpha_{1}, \alpha_{2}$ such that

$$
\begin{gathered}
\|f(x(k), p(x(k)+e(k)))\| \leq L\|x(k)\|+L\|e(k)\| \\
\alpha_{1}(\|x\|) \leq W(x) \leq \alpha_{2}(\|x\|) \quad \forall x \in \mathbb{R}^{n} \\
W(f(x(k), p(x(k)+e(k))))-W(x(k)) \leq \\
-a W(x(k))+\gamma\|e(k)\| \\
\alpha_{1}^{-1}(\|x\|) \leq L_{1}\|x\|
\end{gathered}
$$

Let $k_{i}$ be the last update time. For each $k \in\left[k_{i}, k_{i+1}\right)$, we can then compute $e(k+1)=x\left(k_{i}\right)-x(k+1)$, so that $\|e(k+1)\| \leq\|x(k+1)\|$, and thus $\|e(k+1)\| \leq L\|x(k)\|+$ $L\|e(k)\|$. Further note that $x(k)=x\left(k_{i}\right)-e(k)$, so that $\|e(k+1)\| \leq 2 L\|e(k)\|+L\left\|x\left(k_{i}\right)\right\|$. Recalling that $e\left(k_{i}\right)=0$, the comparison principle for discrete-time systems (see for example, Proposition 1 in [3]) yields

$$
\|e(k)\| \leq \frac{(2 L)^{k}-1}{2(2 L-1)}\left\|x\left(k_{i}\right)\right\|
$$

for all $k \in\left[k_{i}, k_{i+1}\right)$. Equation (28) then yields

$$
\begin{gathered}
W(f(x(k), p(x(k)+e(k))))-W(x(k)) \leq \\
-a W(x(k))+\gamma \frac{(2 L)^{k}-1}{2(2 L-1)}\left\|x\left(k_{i}\right)\right\|
\end{gathered}
$$

From (27), (29) we also have

$$
\left\|x\left(k_{i}\right)\right\| \leq \alpha_{1}^{-1}\left(\left\|W\left(x\left(k_{i}\right)\right)\right\|\right) \leq L_{1} W\left(x\left(k_{i}\right)\right)
$$

Denoting $\psi(k)=\gamma \frac{(2 L)^{k}-1}{2(2 L-1)} L_{1}$ we get $W(x(k+1)) \leq(1-$ a) $W(x(k))+\psi(k) W\left(x\left(k_{i}\right)\right)$. Using again the comparison principle of [3] and assuming $a<1$, we get

$$
W(x(k)) \leq \frac{1-(1-a)^{k}}{a(1-a)} \psi(k) W\left(x\left(k_{i}\right)\right)
$$

Similarly to [22], assume that events are triggered according to

$$
W(x(k))=-\xi W\left(x\left(k_{i}\right)\right)\left(k-k_{i}\right)+W\left(x\left(k_{i}\right)\right)
$$

the right hand side equation is strictly decreasing for $\xi>0$ and thus convergence is guaranteed. Define now the minimum $k=k^{* * *}$ as follows

$$
\begin{aligned}
k^{* * *}= & \arg \min _{k \in \mathbb{N}}\left\{-\xi\left(k-k_{i}\right)+1 \geq\right. \\
& \left.\frac{1-(1-a)^{k}}{a(1-a)} \psi(k)\right\}
\end{aligned}
$$

Then, using (32), (33), a sufficient condition for a nontrivial interexecution time is given by $k^{* * *}>1$. Note that in this case the result holds only for the restricted class of nonlinear systems satisfying Assumption 1.

\section{Event-triggered Model Predictive Control}

In this section, we provide initial results on the main motivation behind the study of event-driven strategies for discrete-time systems, namely, the application on computing the inter-sample times in a Model Predictive Control framework.

Consider that the feedback control that we use to stabilize the plant is computed with a Model Predictive Control (abbr. MPC) formulation. It is widely known that this approach is computationally demanding, in the sense that at each sampling period a finite-time optimal control problem must be solved. In this paper we propose an alternative approach based on the event-triggered framework described previously that may be used to reduce the computational load of the MPC framework.

MPC is an implicit feedback policy, and thus, the eventtrigger condition defined in (16), cannot be directly used. In order to reach in an MPC event-trigger policy, we will use the results as well as the notation of [9], where ISS properties of linear MPC were investigated. Specifically, [9] deals with linear systems and it is proven that the closed-loop system with a receding horizon feedback is globally ISS, when the system is open-loop stable and when input constraints are present. In the case of unstable system, though, the same results apply, but have local nature.

We consider the same scheme as in (11). We assume that the prediction horizon is $N$. The solution of the optimization MPC problem is the optimal sequence

$$
u^{o}(x)=\left\{u^{o}(0 ; x), u^{o}(1 ; x), \ldots, u^{o}(N-1 ; x)\right\}
$$

Consider a set $X_{f}$, over which there exist a feasible and stabilizing control, and thus, application of this feasible controller results in feasible state trajectories. Consider, also, a controllability set $X_{n}$, i.e., the set of all initial conditions that can be steered into the set $X_{f}$ in $N$ steps or less, where the MPC feedback controller is defined. The optimization problem has the cost function

$$
\begin{aligned}
V_{N}^{*}(x) & \left.=\min \sum_{i=0}^{N-1}\left(x(k)^{T} Q x(k)+u^{T}(k) R u(k)\right)\right) \\
& +F(x(N))
\end{aligned}
$$


where $Q>0$ and $R>0$ are appropriate performance functions. With particular choices of the terminal state function $F(\cdot)$ and the set $X_{f}$, it can be proved that the open-loop stable system (11), under the receding horizon feedback $\kappa_{N}(x):=u^{o}(0 ; x)$ can be rendered exponentially stable. The closed-loop system is a piecewise affine system which is stable with a piecewise and differentiable quadratic Lyapunov function $V_{N}^{*}(x)=\bar{x}^{T} P_{i(x)} \bar{x}$, where $\bar{x}=\left[\begin{array}{ll}x & 1\end{array}\right]$, and $i(\cdot)$ is a switching function that maps the state space to a finite set of indices labelling the polytopic partitions of the state space.

In [9], the authors proved that the receding horizon scheme globally ISS stabilizes stable linear systems with input constraints, with respect to additive disturbance. In an eventtriggered formulation, the error, defined as the difference in (6), can be considered as the additive disturbance. Thus the closed-loop system becomes

$$
\begin{aligned}
x(k+1) & =\left(A-B \kappa_{N}(x)\right) x(k)+B \kappa_{N}(x) e(k) \\
& :=\bar{A}_{i(x)} x(k)+B \kappa_{N}(x) e(k)
\end{aligned}
$$

where $\bar{A}_{i(x)}$ is the closed-loop matrix corresponding to the $i$ th partition of the state space, and $i$ is the switching function that maps the state space to a finite set of indices corresponding to different polytopic regions where the active constraints do not change.

Constant sampling implies a zero error and global exponential stability for the closed-loop system

$$
V_{N}^{*}\left(A_{i(x)} x\right)-V_{N}^{*}(x) \leq-C_{q}\|x\|^{2}
$$

where $C_{q}>0$ is the rate of the exponential decay. The differentiability of the Lyapunov function implies

$$
\left\|\frac{V_{N}^{*}(x)}{x}\right\|:=\left\|P_{i(x)} x\right\| \leq \tilde{L}\|x\|
$$

where $\tilde{L}:=\max _{i} \lambda_{\max }\left(P_{i}\right)$. The maximum is taken over all possible partitions, and $\lambda_{\max }$ is the largest singular value of $P_{i}$. Following a similar procedure as in [9], we obtain the following result, which shows that the Lyapunov function defined in (35), is also an ISS Lyapunov function

$$
\begin{aligned}
V_{N}^{*}(x(k+1))-V_{N}^{*}(x(k)) & \leq\left(-C_{q}+\epsilon \tilde{L} C_{A}\right)\|x(k)\|^{2} \\
& +\left(1+\frac{1}{\epsilon}\right) \tilde{L} C_{A} C_{B}\|e(k)\|^{2}
\end{aligned}
$$

where $C_{A}:=\max _{i}\left\|\bar{A}_{i(x)}\right\|$ and we let $C_{B}$, be $C_{B}:=$ $\left\|B \kappa_{N}(x)\right\|^{2}$. Thus we reach to a conclusion for the eventtriggered formulation of a model predictive control system, stated below

Theorem 4: The controller updates with event-triggered formulation for a linear system as (11) under receding horizon control, can be implemented when

$$
\|e(k)\|^{2} \leq \Theta\|x(k)\|^{2}
$$

is violated,where

$$
\Theta=\frac{\left(1+\frac{1}{\epsilon}\right) \tilde{L} C_{A} C_{B}}{\sigma\left(C_{q}-\epsilon \tilde{L} C_{A}\right)}
$$

and $0<\sigma<1$.

\section{EXAMPLES}

In this section we provide some simulation results in order to assess the efficiency of the proposed event-triggered, as well as self-triggered stabilizing controllers in the linear case.

The process we consider is a linear, unstable, discrete-time system described by

$$
x(k+1)=A x(k)+B u(k)
$$

where matrix $A=\left[\begin{array}{cc}0.1 & 1.2 \\ 0.007 & 1.05\end{array}\right]$ and matrix $B=$ $\left[\begin{array}{cc}300 & 200 \\ 0.5 & 0.001\end{array}\right]$. The control sequence is considered to be optimal and can be determined from an LQR problem, which minimizes a cost function of the form

$$
J(u)=\sum_{k=0}^{\infty}\left[x^{T}(k) Q x(k)+u^{T}(k) R u(k)\right]
$$

with performance matrices $Q=\left[\begin{array}{cc}0.001 & 0 \\ 0 & 0.001\end{array}\right]$ and $R=$ $\left[\begin{array}{ll}0.01 & 0.01 \\ 0.01 & 0.01\end{array}\right]$. The linear state feedback control law is written in analytical form as $u^{*}(k)=K x(k)$, where the matrix $K$ is given by $K=-\left[R+B^{T} P B\right]^{-1} B^{T} P A$. The matrix $P$ is the unique, symmetric, and positive-definite solution of the discrete-time algebraic Riccati equation $P=A^{T} P(A+$ $B K)+Q$. We are going to use $P$ in the quadratic ISSLyapunov equation, with $V(x)=x^{T} P x$ being the Lyapunov function candidate. We also define another matrix $\tilde{Q}$ which satisfies the following equation

$$
P-(A+B K)^{T} P(A+B K)=\tilde{Q}
$$

For the particular problem (41) and the event-trigger policy given in (16) we choose $\sigma=0.98$. Then the constant $\mu$ has the value $\mu=0.2934$. Assume, also, that the initial state conditions are $x_{0}=[-0.2,0.5]^{T}$ and that we want to stabilize system (41) at the equilibrium.

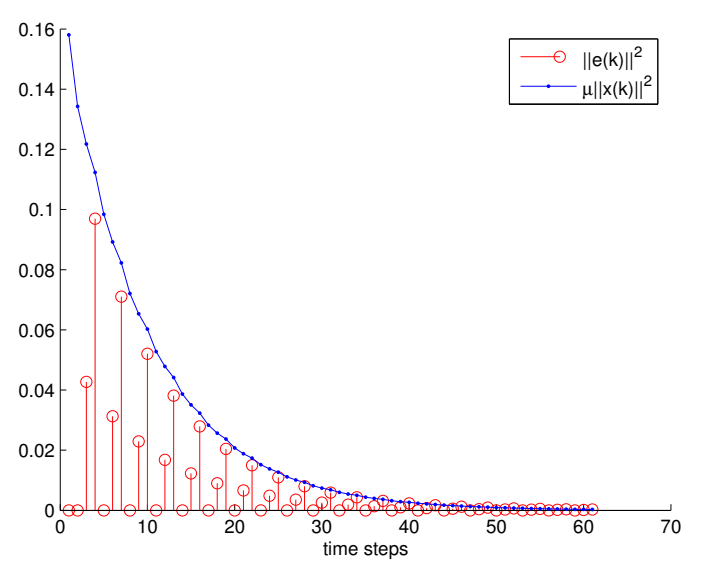

Fig. 1. Evolution of the error norm in the event-triggered case. Red stems represent the evolution of the error norm $\|e(k)\|$ which stays below the state-dependent threshold $\mu\|x(k)\|$ which is represented by the blue line in the Figure. 
Figure 1 depicts the norm of the error $\|e(k)\|$. This stays below the specified state-depended threshold, as given in (16) and is represented by the blue solid line in the Figure. It can be witnessed that using this event-trigger policy, which is conservative, we can sample in periodic fashion, every three steps.

The next Figure depicts the sampling of system (41) under the self-triggered framework. In order to better visualize when sampling takes place, under the self-trigger policy, Figure 2, depicts the difference

$$
D:=\|y(k)\|^{2}-\left(1+\mu^{2}\right)\|\tilde{I} y(k)\|^{2}
$$

where we used (23). When $D$, represented by the blue stems, is below zero, there is no need for sampling, or in other words, there is sampling when the blue stems are above the zero line.

From the simulations is apparent that the system converges under the event-triggered and self-triggered control frameworks. The periodicity that takes place in both cases, is a topic of the ongoing research.

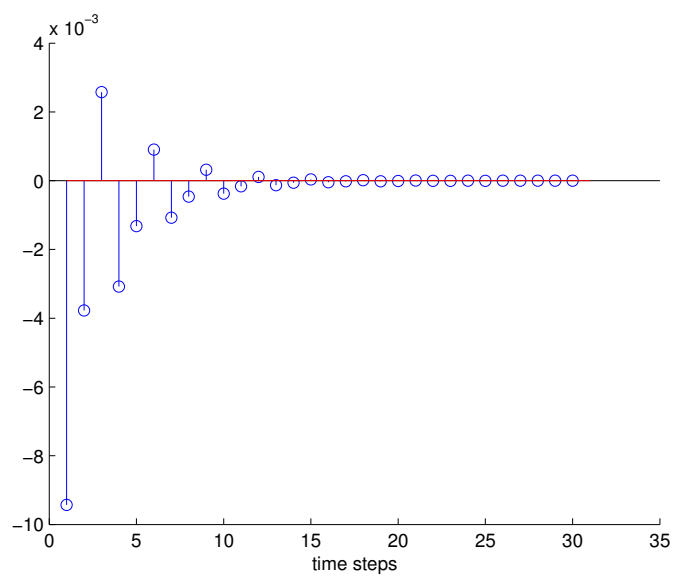

Fig. 2. Blue stems represent the difference given in (44).

\section{CONCLUSIONS}

In this paper, event-triggered strategies for control of discrete-time systems were proposed and analyzed. Similarly to the continuous-time case, the plant is assumed inputto-state stable with respect to measurement errors and the control law is updated once a triggering condition involving the norm of a measurement error is violated. We considered both nonlinear and linear plant and sufficient condition for non-trivial inter-execution times were derived. The results were also extended to a self-triggered formulation, where the next control updates are decided at the previous ones, thus relaxing the need for continuous monitoring of the measurement error. The overall framework was then used in a novel Model Predictive Control approach. The results were illustrated through simulated examples.

Future research will involve further integration of the event-triggered approach with the model predictive control framework. In particular, the ISS property of MPC algorithms will be investigated both for continuous and discretetime systems and will be integrated with the event-triggered framework in a more computationally efficient framework.

\section{REFERENCES}

[1] A. Anta and P. Tabuada. To sample or not to sample: self-triggered control for nonlinear systems. IEEE Transactions on Automatic Control, 2009. to appear.

[2] K.J. Astrom and B. Bernhardsson. Comparison of Riemann and Lebesgue sampling for first order stochastic systems. 41st IEEE Conference on Decision and Control, pages 2011-2016, 2002.

[3] G. Bitsoris and E. Gravalou. Comparison principle, positive invariance and constrained regulation of nonlinear systems. Automatica, 31(2):217-222, 1995.

[4] D.V. Dimarogonas and K.H. Johansson. Event-triggered control for multi-agent systems. 48th IEEE Conf. Decision and Control, 2009. to appear.

[5] D.V. Dimarogonas and K.H. Johansson. Event-triggered cooperative control. European Control Conference, 2009. to appear.

[6] W.P.M.H. Heelmes, J.H. Sandee, and P.P.J. Van Den Bosch. Analysis of event-driven controllers for linear systems. International Journal of Control, 81(4):571-590, 2007.

[7] S. Huang, M.R. James, D. Nesic, and P.M. Dower. Analysis of input-to-state stability for discrete-time nonlinear systems via dynamic programming. Automatica, 41:2055-2065, 2005.

[8] Y. Iino, T. Hatanaka, and M. Fujita. Event-predictive control for energy saving of wireless networked control system. American Control Conference, pages 2236-2242, 2009

[9] A. Jadbabaie and A.S. Morse. On the ISS property for receding horizon control of constrained linear systems. IFAC World Congress, 2002.

[10] J.P. Jiang, E.D. Sontag, and Y. Wang. Input-to-state stability for discrete-time nonlinear systems. IFAC World Congress, pages 277282, 1999.

[11] Z.-P. Jiang and Y. Wang. Input-to-state stability for discrete-time nonlinear systems. Automatica, 37(6):857-869, 2001.

[12] M. Mazo Jr., A. Anta, and P. Tabuada. On self-triggered control for linear systems: Guarantees and complexity. European Control Conference, 2009.

[13] M. Mazo Jr. and P. Tabuada. On event-triggered and self-triggered control over sensor/actuator networks. 47th IEEE Conf. Decision and Control, pages 435-440, 2008.

[14] H. Khalil. Nonlinear Systems. Prentice-Hall, 2002.

[15] D. Lehmann and J. Lunze. Event-based control: A state feedback approach. European Control Conference, pages 1716-1721, 2009.

[16] M. Lemmon, T. Chantem, X.S. Hu, and M. Zyskowski. On selftriggered full-information $\mathrm{H}$-infinity controllers. Hybrid Systems: Computation and Control, 2007.

[17] D. Nesic, A. Teel, and E. Sontag. Formulas relating KL stability estimates of discrete-time and sampled-data nonlinear systems. Systems \& Control Letters, 38:49-60, 1999.

[18] M. Rabi, K.H. Johansson, and M. Johansson. Optimal stopping for event-triggered sensing and actuation. 47th IEEE Conference on Decision and Control, pages 3607-3612, 2008.

[19] E.D. Sontag. Input to state stability: Basic concepts and results. In Nonlinear and Optimal Control Theory, pages 163-220. Springer Berlin / Heidelberg, 2008.

[20] P. Tabuada. Event-triggered real-time scheduling of stabilizing control tasks. IEEE Transactions on Automatic Control, 52(9):1680-1685, 2007.

[21] M. Velasco, J. Fuertes, and P. Marti. The self-triggered task model for real-time control systems. In Work in Progress Proceedings of the 24th IEEE Real-Time Systems Symposium, pages 67-70, 2005.

[22] X. Wang and M.D. Lemmon. Event design in event-triggered feedback control systems. 47th IEEE Conf. Decision and Control, pages 21052110,2008

[23] X. Wang and M.D. Lemmon. Self-triggered feedback control systems with finite-gain L2 stability. IEEE Transactions on Automatic Control, 45(3):452-467, 2009. 